SCIENTIFIC LETTER

\title{
Impact of stroke volume on mitral annular velocities derived from tissue Doppler imaging
}

\author{
C Bruch, J Stypmann, R Gradaus, G Breithardt, T Wichter
}

Heart 2005;91:243-244. doi: 10.1136/hrt.2003.024612

M itral annular velocities derived from pulsed tissue Doppler imaging (TDI) were recently demonstrated to complement the analysis of systolic and diastolic left ventricular (LV) performance in different clinical settings. ${ }^{2}$ The early diastolic mitral annular velocity $\left(E^{\prime}\right)$ was found to behave as a preload independent index of relaxation. ${ }^{3}$ The mitral $\mathrm{E} / \mathrm{E}^{\prime}$ ratio (that is, the mitral $\mathrm{E}$ velocity corrected for the influence of relaxation) has been suggested as an estimate of LV filling pressures. ${ }^{3}$

We hypothesised that LV stroke volume (SV) impacts on mitral annular velocities derived from TDI. Thus, mitral annular velocities should be altered in the presence of either increased or reduced SV.

\section{METHODS}

LV stroke volume index (SVI) was derived from biplane two dimensional images by subtracting LV end systolic volume from LV end diastolic volume, calculated by using Simpson's rule. According to previously published normal values, ${ }^{4}$ expressed as mean (SEM), an SVI of 35.0 (6.8) $\mathrm{ml} / \mathrm{m}^{2}$ (range $22-48 \mathrm{ml} / \mathrm{m}^{2}$ ) was considered normal for female subjects, and an SVI of $39.9(6.0) \mathrm{ml} / \mathrm{m}^{2}$ (range $28-52 \mathrm{ml} / \mathrm{m}^{2}$ ) was considered normal for male subjects. Following these definitions, three study groups were separated. Group 1 comprised 19 patients with increased SVI (mean 62 (18) ml/ $\mathrm{m}^{2}$ ), mean age 53 years (range $36-85$ years), 12 with primary mitral regurgitation, (mean grade $2.9(0.5))$, and seven with aortic regurgitation (mean grade $2.6(0.5)$ ). Group 2 comprised 41 patients with reduced SVI (19 (5) $\left.\mathrm{ml} / \mathrm{m}^{2}\right)$, mean age 60 years, (range $42-85$ years), 27 with underlying coronary artery disease, nine with dilated cardiomyopathy, and five with hypertensive heart disease. Twenty nine asymptomatic age matched subjects with normal SVI served as a control group (SVI $36(9) \mathrm{ml} / \mathrm{m}^{2}$, mean age 55 years (range 40-76 years)). Patients with atrial fibrillation, single or dual chamber pacemakers, or bundle branch block were excluded.

Conventional echo images were taken according to the guidelines of the American Society of Echocardiography. ${ }^{5}$ For TDI recordings, from the apical window peak systolic mitral annular velocity $\left(S^{\prime}\right), E^{\prime}$, and late diastolic annular velocity $\left(\mathrm{A}^{\prime}\right)$, were obtained from four positions (septal, lateral, anterobasal, and inferior sites of the mitral annulus) and averaged.

\section{RESULTS}

The two study groups and the control group did not differ significantly with respect to age, heart rate, body surface area, or systolic/diastolic blood pressure. Patients with reduced SV were in a poorer New York Heart Association (NYHA) functional class than those with increased SV. In both groups, LV end diastolic diameters were increased in comparison to controls. In those with reduced SV, LV end systolic diameters and end systolic volumes were elevated, and ejection fraction and fractional shortening were reduced.
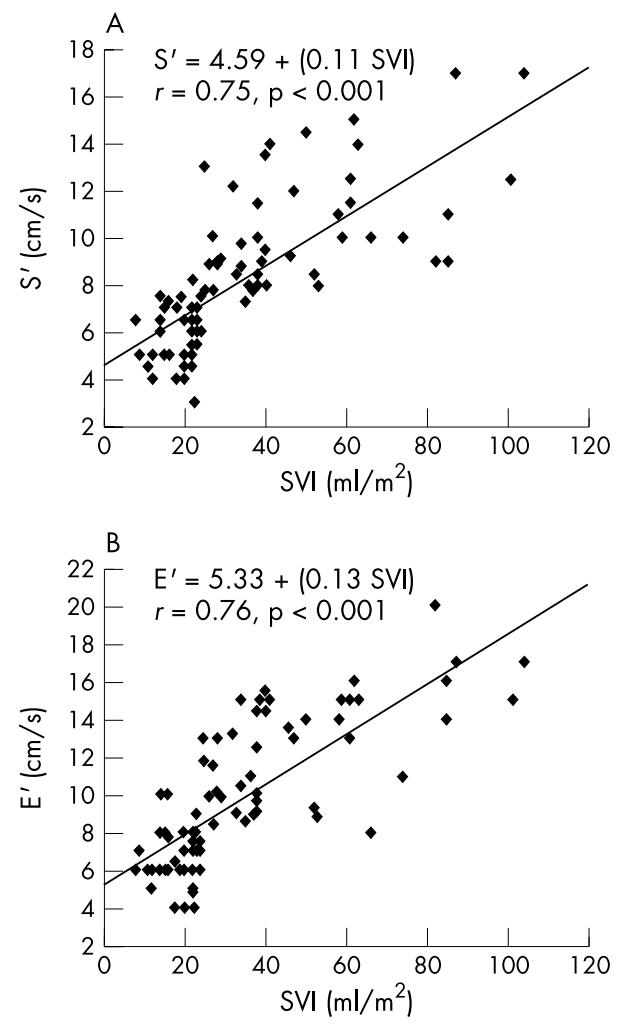

Figure 1 (A) Scatter plot of the relation between SVI and $S^{\prime}$. (B) Scatter plot of the relation between SVI and $\mathrm{E}^{\prime}$.

No significant differences between study groups were observed with respect to mitral A velocity, E/A ratio, deceleration time and isovolumic relaxation time.

TDI analysis of mitral annulus velocity was readily obtained in all study subjects. S' was higher in patients with increased SV $(12.0(2.2) \mathrm{cm} / \mathrm{s})$ in comparison to controls $(8.8$ (1.3) $\mathrm{cm} / \mathrm{s})$, but lower in patients with reduced SV $(5.7$ (1.2) $\mathrm{cm} / \mathrm{s})($ all $\mathrm{p}<0.01)$. E' was also increased in patients with increased SV $(14.1(2.7) \mathrm{cm} / \mathrm{s})$ in comparison to controls ( $11.6(2.5) \mathrm{cm} / \mathrm{s})(\mathrm{p}<0.05)$, but reduced in patients with reduced SV $(6.7(1.5) \mathrm{cm} / \mathrm{s})(\mathrm{p}<0.001$, reduced SV $v$ control/ increased SV groups). On simple linear regression analysis, SVI was significantly related to $S^{\prime}(r=0.75, \mathrm{p}<0.0001$; fig lA), to $\mathrm{E}^{\prime}(r=0.76, \mathrm{p}<0.0001$, fig $1 \mathrm{~B})$ and to $\mathrm{A}^{\prime}$ $(r=0.43, \mathrm{p}<0.01)$. On multiple regression analysis, SVI was a stronger independent predictor of $S^{\prime}$ and $E^{\prime}$ than conventional systolic or diastolic echocardiographic variables.

Abbreviations: $A^{\prime}$, late diastolic mitral annular velocity; $E^{\prime}$, early diastolic mitral annular velocity; $\mathrm{S}^{\prime}$, peak systolic mitral annular velocity; $\mathrm{SVI}$, stroke volume index 
Intra-observer and inter-observer variability for conventional two dimensional Doppler measurements and TDI derived parameters of mitral annulus velocity $\left(\mathrm{S}^{\prime}, \mathrm{E}^{\prime}, \mathrm{A}^{\prime}\right.$, $\left.E^{\prime} / A^{\prime}\right)$ ranged from 3-9\%.

\section{DISCUSSION}

Mitral annular velocities derived from TDI have recently been demonstrated to provide relevant complementary information in a variety of cardiac disorders. ${ }^{12}$ With changes in volume status, changes in the annular or myocardial tissue velocities have been noted to be less than the corresponding changes in mitral flow velocities. ${ }^{2}$ This has led to the conclusion that annular/myocardial velocities are relatively volume independent. Consequently, $\mathrm{E}^{\prime}$ was proposed as an index of $\mathrm{LV}$ relaxation, and the mitral $\mathrm{E} / \mathrm{E}^{\prime}$ ratio was considered as an estimate of LV filling pressures in subjects with preserved and reduced ejection fraction. ${ }^{3}$ Our study is the first to demonstrate that LV SV has a significant impact on mitral annular velocities derived from TDI. In subjects with increased SV caused by significant mitral or aortic regurgitation, $\mathrm{S}^{\prime}$ and $\mathrm{E}^{\prime}$ were significantly increased as compared to healthy controls. In contrast, in subjects with reduced SV because of ischaemic cardiomyopathy, dilated cardiomyopathy, or hypertensive heart disease, $S^{\prime}$ and $E^{\prime}$ were significantly decreased. Using linear regression analysis, SV related significantly with $S^{\prime}$ and $E^{\prime}$, with $r$ values of 0.75 and 0.76 (fig 1), and to a lesser degree with $\mathrm{A}^{\prime}$, respectively. Most importantly, on multiple regression analysis SV was a stronger independent predictor of $S^{\prime}$ and $E^{\prime}$ than conventional systolic or diastolic parameters.

Our findings may have important implications with respect to the interpretation of mitral annular velocities recorded in different subsets of patients. In subjects with impaired systolic/diastolic performance and concomitant mitral or aortic regurgitation, a significant increase in volume flow across the mitral or aortic valve may result in notably increased $S^{\prime}$ and $E^{\prime}$. Thus, the presence of abnormal contraction/relaxation may be masked in the annular velocity tracing derived from TDI. In these patients, the $\mathrm{E} / \mathrm{E}^{\prime}$ ratio is likely to be reduced and may be misleading as an estimate of filling pressures.

This study excluded patients with atrial fibrillation, single or dual chamber pacemakers, or bundle branch block, and so the results should not be extrapolated to these patient populations. Annular velocities were measured in subjects with increased SV caused by significant mitral or aortic regurgitation. Thus, we are not sure that our results can be applied to patients with increased SV for other reasons such as fever, anaemia, or septicaemia.

\section{Authors' affiliations}

C Bruch, J Stypmann, R Gradaus, G Breithardt, T Wichter, Department of Cardiology and Angiology, Hospital of the University of Muenster, Muenster, Germany

Correspondence to: Dr Christian Bruch, Medizinische Klinik und Poliklinik C, Universitätsklinikum Münster, Albert-Schweitzer-Strasse 33, D-48149 Münster, Germany; bruchc@uni-muenster.de

Accepted 25 March 2004

\section{REFERENCES}

1 Rodriguez L, Garcia M, Ares M, et al. Assessment of mitral annular dynamics during diastole by Doppler tissue imaging: comparison with mitral Doppler inflow in subjects without heart disease and in patients with left ventricular hypertrophy. Am Heart J 1996;131:982-7.

2 Sohn DW, Chai ICH, Lee DJ, et al. Assessment of mitral annulus velocity by Doppler tissue imaging in the evaluation of left ventricular diastolic function. J Am Coll Cardiol 1997;30:474-80.

3 Nagueh SF, Bachinski LL, Meyer D, et al. Tissue Doppler imaging consistently detects myocardial abnormalities in patients with hypertrophic cardiomyopathy and provides a novel means for an early diagnosis before and independently of hypertrophy. Circulation 2001;104:128-30.

4 Erbel R, Henkel B, Ostländer C, et al. Normalwerte für die zweidimensionale Echokardiographie. Deutsch Med Wochenschr 1985;1 10:123-8.

5 Schiller NB, Shah PM, Crawford M, et al. Recommendations for quantification of the left ventricle by two-dimensional echocardiography. J Am Soc Echocardiogr 1989;2:358-67.

\section{IMAGES IN CARDIOLOGY}

A 36 year old man was admitted with a four week history of worsening exertional dyspnoea and peripheral oedema. Transoesophageal echocardiography (TOE) revealed a ruptured aneurysm of the non-coronary sinus of Valsalva (SVA) protruding into the right atrium with a significant systemic to pulmonary shunt associated with enlargement of the right atrium and right ventricle. There was also an incidental finding of an interatrial septal aneurysm and patent foramen ovale (panel A). The patient underwent percutaneous closure of the ruptured sinus of Valsalva aneurysm using a $14 \mathrm{~mm}$ Amplatzer septal occluder device (panel B). This achieved a good result with a rapid improvement in the patient's symptoms. There was only a mild residual shunt seen on TOE following the closure (panel C).

Transcatheter closure can be an effective treatment for a ruptured sinus of Valsalva aneurysm in selected cases.
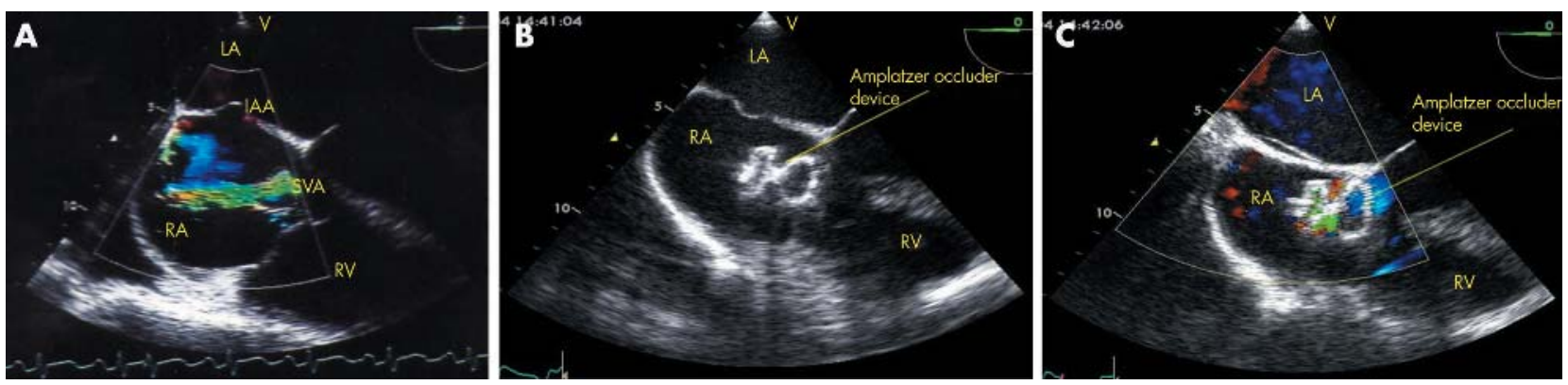